\title{
The impact of temporary clipping during aneurysm surgery on the incidence of delayed cerebral ischemia after aneurysmal subarachnoid hemorrhage
}

\author{
Vesna Malinova, MD, ${ }^{1}$ Bawarjan Schatlo, MD, ${ }^{1}$ Martin Voit, ${ }^{1}$ Patricia Suntheim, ${ }^{1}$ Veit Rohde, MD, ${ }^{1,2}$ \\ and Dorothee Mielke, MD'
} 1Department of Neurosurgery, Georg-August-University Göttingen; and 2Department of Neurosurgery, RWTH University,
Aachen, Germany

\begin{abstract}
OBJECTIVE Clipping of a ruptured intracranial aneurysm requires some degree of vessel manipulation, which in turn is believed to contribute to vasoconstriction. One of the techniques used during surgery is temporary clipping of the parent vessel. Temporary clipping may either be mandatory in cases of premature rupture (rescue) or represent a precautionary or facilitating surgical step (elective). The aim of this study was to study the association between temporary clipping during aneurysm surgery and the incidence of vasospasm and delayed cerebral ischemia after aneurysmal subarachnoid hemorrhage (aSAH) in a large clinical series.
\end{abstract}

METHODS Seven hundred seventy-eight patients who underwent surgical aneurysm treatment after aSAH were retrospectively included in the study. In addition to surgical parameters, the authors recorded transcranial Doppler (TCD) sonography-documented vasospasm (TCD-vasospasm, blood flow acceleration $>120 \mathrm{~cm} / \mathrm{sec}$ ), delayed ischemic neurological deficits (DINDs), and delayed cerebral infarction (DCI). Multivariate binary logistic regression analysis was applied to assess the association between temporary clipping, vasospasm, DIND, and DCl.

RESULTS Temporary clipping was performed in 338 (43.4\%) of 778 patients during aneurysm surgery. TCD sonographic flow acceleration developed in 370 (47.6\%), DINDs in $123(15.8 \%)$, and DCl in 97 (12.5\%). Patients with temporary clipping showed no significant increase in the incidence of TCD-vasospasm compared with patients without temporary clipping ( $49 \%$ vs $48 \%$, respectively; $p=0.60$ ). DINDs developed in $12 \%$ of patients with temporary clipping and $18 \%$ of those without temporary clipping $(p=0.01)$. $\mathrm{DCl}$ occurred in $9 \%$ of patients with temporary clipping and $15 \%$ of those without temporary clipping ( $p=0.02)$. The need for rescue temporary clipping was a predictor for $\mathrm{DCl} ; 19.5 \%$ of patients in the rescue temporary clipping group but only $11.3 \%$ in the elective temporary clipping group had infarcts $(p=0.02)$. Elective temporary clipping was not associated with TCD-vasospasm $(p=0.31)$, DIND $(p=0.18)$, or DCI $(p=0.06)$.

CONCLUSIONS Temporary clipping did not contribute to a higher rate of TCD-vasospasm, DIND, or DCI in comparison with rates in patients without temporary clipping. In contrast, there was an association between temporary clipping and a lower incidence of DINDs and $\mathrm{DCl}$. There is no reason to be hesitant in using elective temporary clipping if deemed appropriate.

https://thejns.org/doi/abs/10.3171/2017.3.JNS162505

KEY WORDS subarachnoid hemorrhage; cerebral vasospasm; temporary clipping; aneurysm surgery; vascular disorders

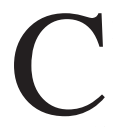
EREBRAL vasospasm associated with delayed ischemic neurological deficits (DINDs) and/or delayed cerebral infarction (DCI) is one of the most severe complications after aneurysmal subarachnoid hemorrhage (aSAH), and it leads to severe disability or mortality in
$38 \%$ of cases. ${ }^{3,9,14,28}$ Cerebral vasospasm proven by digital subtraction angiography (DSA) occurs in 30\%-70\% of patients suffering from aSAH. ${ }^{31,32}$ DINDs affect about $25 \%-30 \%$ of all patients with aSAH, and $3 \%-7 \%$ of these patients develop vasospasm-induced DCIs. ${ }^{10,30}$ The patho-

ABBREVIATIONS aSAH = aneurysmal subarachnoid hemorrhage; $\mathrm{DCl}=$ delayed cerebral infarction; $\mathrm{DIND}=$ delayed ischemic neurological deficit; $\mathrm{DSA}=$ digital subtraction angiography; $T C D=$ transcranial Doppler; TCD-vasospasm = TCD sonography-documented vasospasm; WFNS = World Federation of Neurosurgical Societies. SUBMITTED September 29, 2016. ACCEPTED March 10, 2017.

INCLUDE WHEN CITING Published online September 15, 2017; DOI: 10.3171/2017.3.JNS162505. 
genesis of DCI after aSAH is assumed to be of a multifactorial nature. Different risk factors have been described for the development of cerebral vasospasm and DCI after aSAH. $., 15,24,25,31,32$ Both endovascular catheter placement for coiling of the aneurysm and aneurysm clipping require vessel manipulation. This manipulation results in vasoconstriction and potentially prolonged or delayed vasospasm.

In premature aneurysm rupture, rescue temporary clipping of the parent vessel stops the arterial bleeding, a prerequisite for successful aneurysm clipping. Elective temporary clipping of the parent vessel is used for safe exposure of the aneurysm-artery complex. Moreover, temporary clipping allows larger aneurysms to soften, facilitating visualization of otherwise hidden structures. ${ }^{7}$ In experimental studies, decreased endothelium-dependent vasorelaxation response and transformation in the tunica media have been described after temporary clipping..$^{20,22}$ Additionally, an experimental study showed a significant reduction in tissue perfusion after temporary clipping, with the extent of perfusion deficit depending on the extent of collateral blood supply. ${ }^{4}$ Temporary clipping during aneurysm surgery may potentially further enhance the vulnerability of the cerebral vasculature to vasospasm. We therefore generated the hypothesis that temporary clipping-either planned or after premature aneurysm rupture-increases the risk for cerebral vasospasm and DCI in patients with aSAH undergoing aneurysm surgery. The aim of this study was to evaluate the impact of temporary clipping during aneurysm surgery on the incidence of transcranial Doppler (TCD) sonography-documented vasospasm (TCD-vasospasm), DINDs, and DCI after aSAH.

\section{Methods}

All procedures followed were in accordance with the ethical standards of the responsible committees on human experimentation (institutional and national) and with the Helsinki Declaration of 1975, as revised in 2008. ${ }^{33}$

\section{Patient Population}

We retrospectively analyzed a database that included 1338 patients suffering from aSAH. The patient data were retrospectively collected for the period from 1989 to 1996, prospectively collected from 1997 to 2004, and again retrospectively collected for the period from 2005 to 2014 . To answer the question at hand, we excluded all endovascular cases. A total of 778 consecutive patients were finally included in the study. The development of TCDvasospasm, DINDs, and DCI was documented. The initial clinical presentation of the patients was documented using the Glasgow Coma Scale score and the WFNS (World Federation of Neurosurgical Societies) grading system. The patients' age and sex and the location of the ruptured aneurysms were documented as well. Aneurysm location was dichotomized as anterior circulation (anterior cerebral artery, anterior communicating artery, internal carotid artery, or middle cerebral artery) or posterior circulation (posterior communicating artery, posterior cerebral artery, basilar artery, vertebral artery, or posterior inferior cerebellar artery).

\section{Treatment Protocol}

All patients were treated according to our institutional standard protocol for the management of aSAH. These treatment modalities remained the same during the whole study period; the only change in the overall protocol during the study period was the addition of CT-perfusion imaging, as a diagnostic imaging modality, during the last 2 years. All patients underwent initial CT to confirm the diagnosis of aSAH as well as CT angiography and/or 4-vessel DSA for aneurysm verification. Patients without verified aSAH were excluded from this study. Four hours after surgery, a CT scan was routinely performed to rule out surgery-associated bleeding or infarction. Another CT scan was performed if vasospasm was suspected on clinical grounds or TCD sonography findings. In unconscious or sedated patients, we routinely performed CT on Day 10 after hemorrhage. The management included daily TCD monitoring until Day 14 after hemorrhage. Neurological assessment was performed routinely 3 times a day. Nimodipine was given routinely (usually intravenously) for 14 days. Patients with evidence of cerebral vasospasm were treated with a modified triple- $\mathrm{H}$ therapy, which consisted of induced hypertension (target systolic blood pressure $160 \mathrm{~mm} \mathrm{Hg}$ ) through application of catecholamines (noradrenaline); normovolemia or mild hypervolemia was maintained.

\section{Surgical Technique}

Aneurysm clipping was performed within 48 hours after presentation. For the treatment of aneurysms of the anterior circulation, a pterional craniotomy was performed. The interhemispheric approach was used for aneurysms of the pericallosal artery. Aneurysms of the upper basilar artery were clipped using a subtemporal approach, and aneurysms of the vertebral artery or posterior inferior cerebellar artery were clipped via a transcondylar approach. Intraoperative irrigation with nimodipine was used to reduce the risk of vasospasm due to manipulation of the parent vessel and the surrounding vessels within the operating field, and intraoperative microvascular Doppler sonography was performed to confirm sufficient blood flow in the parent vessels and outgoing branches. Elective temporary clipping was performed at the discretion of the neurosurgeon to facilitate better aneurysm dissection and to avoid intraoperative aneurysm rupture due to the manipulation of the intracranial vessels. Rescue temporary clipping was performed in cases of intraoperative aneurysm rupture.

\section{Primary Outcome Parameters \\ Transcranial Doppler Sonography}

Cerebral vasospasm was diagnosed by TCD sonography and defined by an increase of mean blood flow velocity of $>120 \mathrm{~cm} / \mathrm{sec}$ in the middle cerebral artery. ${ }^{2,3,6}$ TCD sonography measurements were performed on a daily basis over 14 days after aSAH.

\section{Delayed Ischemic Neurological Deficits}

Symptomatic vasospasm was defined as the onset of new global or focal neurological deficits in patients in 
whom rebleeding, hydrocephalus, meningitis, epileptic seizures, or metabolic disorders were ruled out. ${ }^{9}$

\section{Delayed Cerebral Infarction}

Every CT-proven new ischemic lesion after aSAH that was not associated with aneurysm treatment and occurred between Day 4 and Day 14 after aSAH was termed DCI. ${ }^{9}$ A routine CT scan was performed 4 hours after the operation to identify any infarction related to the aneurysm treatment.

\section{Statistical Analysis}

Subgroup specifications are portrayed using descriptive statistics. The 3 outcome parameters were TCD-vasospasm, DIND, and DCI. A chi-square test with Fisher's exact test and a 2-tailed significance level of $p<0.05$ were used to assess whether temporary clip application was associated with any of the outcome variables. All analyses were performed using the statistics software package SPSS version 21.0 (IBM Corp). Missing values were imputed. To evaluate the effect of temporary clipping on outcome parameters (TCD-vasospasm, DIND, and DCI), we performed a multivariate binary logistic regression analysis (including Hosmer-Lemeshow testing). Effect size (odds ratios and 95\% confidence intervals) and $\mathrm{p}$ values were calculated. The potential confounding effects of WFNS grade and Fisher grade were accounted for in the analysis.

\section{Results}

\section{Patient Population}

A total of 778 consecutive patients with aSAH and aneurysm clipping were included in the study. The mean age was 54 years (range 18-87 years). An aneurysm of the anterior circulation was present in 574 (73.8\%) of the 778 patients, and 204 (26.2\%) had an aneurysm of the posterior circulation. The WFNS grade was good (Grades IIII) in 524 cases (67.4\%) and poor (Grade IV or V) in 254 (32.6\%). The Fisher grade was 1 or 2 in 298 cases (38.3\%) and 3 or 4 in 480 (62\%). Patient characteristics are summarized in Table 1.

\section{Primary Outcome Parameters Versus Overall Temporary Clipping}

In 338 (43.4\%) of the 778 patients, temporary clipping was performed during aneurysm surgery. There was an association between overall temporary clipping and high Fisher grade as well as low WFNS grade. However, these results were not statistically significant $(\mathrm{p}=0.41$ and $\mathrm{p}$ $=0.11$, respectively). Temporary clipping was more frequently performed in cases of anterior circulation aneurysm than in posterior circulation aneurysm ( $45 \%$ vs $38 \%$, $\mathrm{p}=0.08)$.

TCD-vasospasm developed in 370 (47.6\%) of 778 patients, DINDs occurred in 123 (15.8\%), and DCI was detected in $97(12.5 \%)$. In the univariate analysis, patients with temporary clipping showed no significant increase in the incidence of TCD-vasospasm compared with patients without temporary clipping ( $49 \%$ vs $48 \%, \mathrm{p}=0.60)$. A high Fisher grade was a predictor of TCD-vasospasm $(\mathrm{p}=$
TABLE 1. Patient population: comparison of the patient groups with and without temporary clipping

\begin{tabular}{|c|c|c|c|c|}
\hline \multirow[b]{2}{*}{ Characteristic } & \multicolumn{3}{|c|}{ Temporary Clipping } & \multirow{2}{*}{$\begin{array}{c}\mathrm{p} \\
\text { value }\end{array}$} \\
\hline & No & Yes & Total & \\
\hline Age $>55$ yrs & & & & 0.580 \\
\hline No & $234(57.5)$ & $173(42.5)$ & $407(100.0)$ & \\
\hline Yes & $206(55.5)$ & $165(44.5)$ & $371(100.0)$ & \\
\hline Male sex & & & & 0.390 \\
\hline No & $297(57.4)$ & $220(42.6)$ & $517(100.0)$ & \\
\hline Yes & $143(54.8)$ & $118(45.2)$ & $261(100.0)$ & \\
\hline High Fisher grade & & & & 0.410 \\
\hline No & $163(54.7)$ & $135(45.3)$ & $298(100.0)$ & \\
\hline Yes & $277(57.7)$ & $203(42.3)$ & $480(100.0)$ & \\
\hline High WFNS grade & & & & 0.110 \\
\hline No & $286(54.6)$ & $238(45.4)$ & $524(100.0)$ & \\
\hline Yes & $154(60.6)$ & $100(39.4)$ & $254(100.0)$ & \\
\hline Anterior circulation & & & & 0.081 \\
\hline No & $126(61.8)$ & 78 (38.2) & $204(100.0)$ & \\
\hline Yes & $314(54.7)$ & $260(45.3)$ & $574(100.0)$ & \\
\hline
\end{tabular}

Data are numbers of patients (\%).

0.02). DIND occurred in $12 \%$ of patients with temporary clipping and $18 \%$ of those without temporary clipping $(\mathrm{p}$ $=0.01)$. Fisher grade was a predictor of DIND $(p<0.001)$. DCI occurred in $9 \%$ of patients with temporary clipping and $15 \%$ of those without temporary clipping $(\mathrm{p}=0.02)$. DCI predictors were Fisher grade $(\mathrm{p}<0.001)$ and WFNS grade $(\mathrm{p}=0.004)$.

In the multivariate analysis, the use of temporary clipping was not associated with the occurrence of TCD-vasospasm (OR 1.10, 95\% CI 0.82-1.47, p = 0.52) but appeared to be inversely associated with the occurrence of DIND (OR 0.42, 95\% CI 0.42-0.97, p = 0.037) and showed a trend toward an inverse association with infarcts (OR 0.64, 95\% CI $0.40-1.02, \mathrm{p}=0.062$ ). The multivariate model accounted for the association of Fisher grade with the occurrence of TCD-vasospasm, DIND, and infarcts. We also included the initial clinical status (WFNS grade) to accommodate the potential confounding role of this important predictor with regard to the effect of temporary clipping. After multivariate correction, Fisher grade was an independent predictor for DIND (OR 2.68, 95\% CI 1.68-4.29, $\mathrm{p}<0.001$ ) and DCI (OR 3.39, 95\% CI 1.94-5.95, $\mathrm{p}<0.001)$ but not for TCD-vasospasm (OR 1.41, 95\% CI 1.05-1.89, $\mathrm{p}=0.21$ ). Poor WFNS grade (IV and V) was predictive of DCI (OR $1.91,95 \%$ CI $1.23-2.96, p<0.01$ ) but not predictive of TCD-vasospasm (OR 1.04, 95\% CI 0.77-1.41, p = 0.81), and it showed a trend toward an association with DINDs (OR 1.47, 95\% CI 0.98-2.19, $\mathrm{p}=0.063$ ). The results of the multivariate analysis are outlined in Fig. 1.

\section{Elective Versus Rescue Temporary Clipping}

Elective temporary clipping was performed in $66.5 \%$ (225/338) of all patients with temporary clipping. Rescue temporary clipping was needed in $33.5 \%$ (113/338) of all patients with temporary clipping. Rescue temporary clip- 


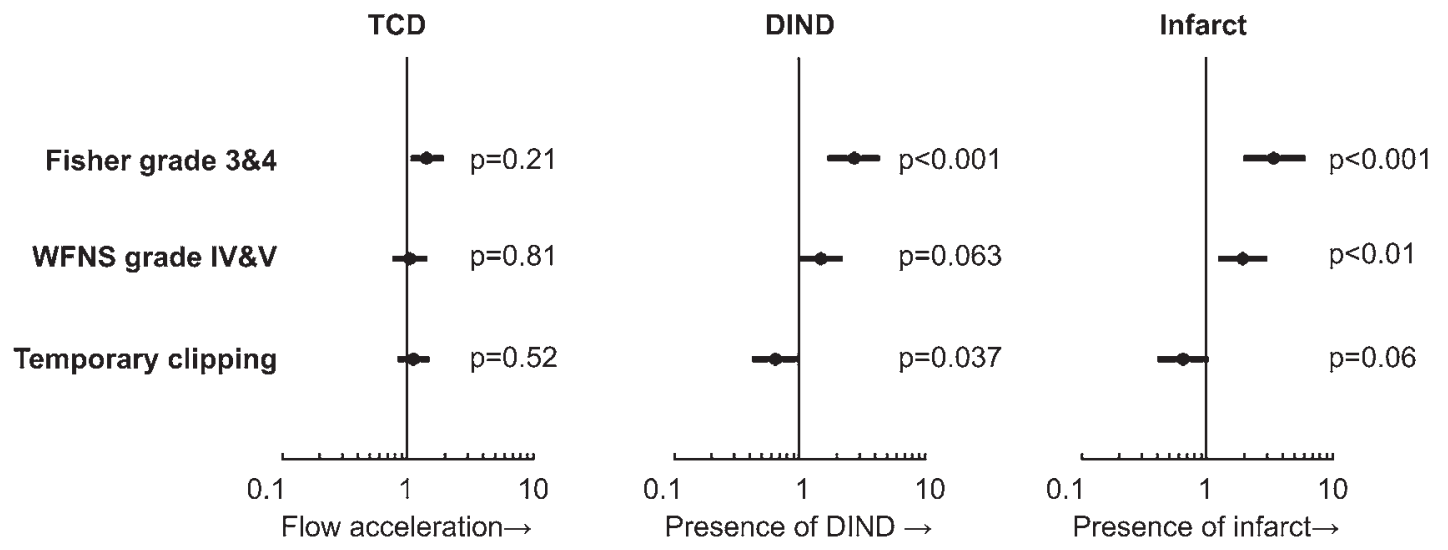

FIG. 1. Multivariate effects of Fisher grade, WFNS grade, and temporary clipping on TCD, DIND, and infarction.

ping was not predictive of TCD-vasospasm (49\% of the patients who had rescue clipping had TCD-vasospasm vs $50 \%$ of the patients who had elective temporary clipping; $\mathrm{p}=0.81)$. In contrast, rescue temporary clipping was a predictor for DINDs $(19.5 \%$ of the patients who had rescue clipping had DINDs vs only $15.2 \%$ of those who did not have temporary clipping). However, this effect was not significant $(\mathrm{p}=0.29)$. Rescue temporary clipping was a predictor for developing DCI: $19.5 \%$ of the patients in the rescue temporary clip group had infarcts, whereas only $11.3 \%$ of the patients not undergoing temporary clipping had infarcts. This difference proved to be significant $(\mathrm{p}=$ 0.02).

In the multivariate analysis, elective temporary clipping was not a predictor of TCD-vasospasm (OR 1.18, 95\% CI 0.85-1.63, $\mathrm{p}=0.31$ ), DIND (OR 0.72, 95\% CI 0.44-1.16, $\mathrm{p}=0.18$ ), or infarction (OR 1.62, 95\% CI 0.98-2.68, $\mathrm{p}=$ 0.06). A high Fisher grade was a predictor of TCD-vasospasm (OR 1.42, 95\% CI 1.03-1.95, p = 0.02), DIND (OR $2.86,95 \%$ CI $1.70-4.81, \mathrm{p}<0.001)$, and infarction (OR $3.41,95 \%$ CI $1.82-6.37, \mathrm{p}<0.001)$. A high WFNS grade was predictive for the occurrence of infarction (OR 1.82, $95 \%$ CI 1.11-3.0, $\mathrm{p}=0.01$ ) but not for TCD-vasospasm (OR 1.11, 95\% CI 0.80-1.54, p = 0.52) or DIND (OR 1.47, 95\% CI 0.94-2.28, $\mathrm{p}=0.08)$.

In the multivariate analysis, rescue temporary clipping was predictive for infarction (OR 1.89, 95\% CI 1.10-3.25, $\mathrm{p}=0.02$ ) but not for DIND (OR 1.32, 95\% CI 0.78-2.22, p $=0.29)$ or TCD-vasospasm (OR 1.05, 95\% CI 0.70-1.57, p $=0.81)$. A high Fisher grade was a predictor of TCD-vasospasm (OR 1.40, 95\% CI 1.04-1.88, p = 0.02), DIND (OR $2.79,95 \%$ CI 1.71-4.35, $\mathrm{p}<0.001$ ), and infarcts (OR 3.43, 95\% CI 1.96-6.02, $\mathrm{p}<0.001)$. A high WFNS grade was a predictor of DIND (OR 1.50, 95\% CI 1.00-2.24, p = 0.04) and infarction (OR 1.96, 95\% CI 1.26-3.05, $\mathrm{p}=0.003$ ) but not TCD-vasospasm (OR 1.03, 95\% CI 0.76-1.40, p $=0.82)$. The results of elective and rescue temporary clipping are summarized in Tables 2 and 3.

\section{Discussion}

The major finding of the present study was that temporary clipping during aneurysm surgery in patients with aSAH does not result in a higher incidence of TCD-va- sospasm, DIND, or DCI. Based on our data, we were not able to identify a detrimental effect of temporary clipping. Therefore, temporary clipping appears to be warranted in selected cases to facilitate aneurysm dissection.

\section{Does Microsurgery Contribute to Vasospasm?}

To what degree surgical manipulation per se is a contributor to delayed vasospasm and DCI in aSAH patients who undergo aneurysm clipping remains a matter of debate. ${ }^{8}$ Many publications in the last 2 decades have suggested that surgery indeed contributes to vasospasm. It is a well-known fact, following the international cooperative study on the timing of aneurysm surgery, ${ }^{18,19}$ that surgery during the peak vasospasm period yields significantly poorer results than early surgery, possibly due to exacerbation of the preexistent vasospasm by manipulation of the parent vessels and their branches. ${ }^{5,16-19,34}$ This clinical finding was supported by imaging studies. Acute angiographic

TABLE 2. Elective temporary clipping versus no temporary clipping

\begin{tabular}{lcccc}
\hline & \multicolumn{3}{c}{ Elective Temporary Clipping } & \multirow{2}{*}{$\begin{array}{c}\text { Total } \\
\text { Value }\end{array}$} \\
\cline { 2 - 4 } Characteristic & Yes & No & & 0.857 \\
\hline Age $>55$ yrs & & & & \\
\hline No & $118(33.5)$ & $234(66.5)$ & $352(100.0)$ & \\
\hline Yes & $107(34.2)$ & $206(65.8)$ & $313(100.0)$ & \\
\hline Male sex & & & & 0.335 \\
\hline No & $144(32.6)$ & $298(67.4)$ & $442(100.0)$ & \\
\hline Yes & $81(36.3)$ & $142(63.7)$ & $223(100.0)$ & \\
\hline High Fisher grade & & & & 0.195 \\
\hline No & $95(36.8)$ & $163(63.2)$ & $258(100.0)$ & \\
\hline Yes & $130(31.9)$ & $277(68.1)$ & $407(100.0)$ & \\
\hline High WFNS grade & & & & 0.088 \\
\hline No & $161(36.0)$ & $286(64.0)$ & $447(100.0)$ & \\
\hline Yes & $64(29.4)$ & $154(70.6)$ & $218(100.0)$ & \\
\hline Anterior circulation & & & & 0.031 \\
\hline No & $47(27.2)$ & $126(72.8)$ & $173(100.0)$ & \\
\hline Yes & $178(36.2)$ & $314(63.8)$ & $492(100.0)$ & \\
\hline
\end{tabular}

Data are numbers of patients (\%). 
TABLE 3. Primary outcome parameters versus rescue temporary clipping

\begin{tabular}{ccccc}
\hline & \multicolumn{3}{c}{ Rescue Temporary Clipping } & \\
\cline { 2 - 4 } Parameter & No & Yes & Total & p Value \\
\hline TCD-vasospasm* & & & & \\
\hline No & $340(85.9)$ & $56(14.1)$ & $396(100.0)$ & 0.814 \\
\hline Yes & $315(85.1)$ & $55(14.9)$ & $370(100.0)$ & \\
\hline DINDs & & & & \\
\hline No & $564(86.1)$ & $91(13.9)$ & $655(100.0)$ & 0.291 \\
\hline Yes & $101(82.1)$ & $22(17.9)$ & $123(100.0)$ & \\
\hline Infarction & & & & \\
\hline No & $590(86.6)$ & $91(13.4)$ & $681(100.0)$ & 0.020 \\
\hline Yes & $75(77.3)$ & $22(22.7)$ & $97(100.0)$ & \\
\hline
\end{tabular}

Data are numbers of patients (\%).

* Data concerning TCD-vasospasm were not available in 12 cases.

vasospasm was reported in about $11 \%$ of patients undergoing aneurysm surgery within the first 72 hours after aSAH, which was associated with the occurrence of vasospasmrelated symptoms and poor outcome.,19 In a series of 109 patients with ruptured $(n=77)$ and unruptured $(n=32)$ aneurysms undergoing clipping or coiling, angiography was performed directly after treatment. Angiographic vasospasm was seen in $30 \%$ of clipped and $14 \%$ of coiled cases. ${ }^{5}$ Treatment-associated vasospasm was more frequent in patients with aSAH than in those with unruptured aneurysms. This could be explained by increased vessel reactivity due to aSAH or by increased vessel manipulation during surgery for ruptured aneurysms. ${ }^{27}$ Another explanation could be a surgery-induced aggravation of early preexisting vasospasm in the patients with ruptured aneurysms. In a retrospective single-center study of 255 patients treated microsurgically $(n=203)$ or endovascularly $(\mathrm{n}=52)$ within 72 hours after aSAH, a significantly higher overall incidence of vasospasm (60\% vs $38 \%$ ) and delayed infarction (17\% vs 6\%) was detected in the patients with clipped aneurysms. ${ }^{13}$ It has to be acknowledged that 2 studies showed no difference between the 2 treatment modalities in aSAH patients..$^{15,30}$ However, even these 2 studies do not necessarily indicate that aneurysm treatment is not associated with an increased risk of therapy-induced or therapy-enhanced vasospasm but only show that the effect is similar irrespective of the treatment modality.

Elective temporary clipping facilitates surgical dissection of the aneurysm and decreases the incidence of intraoperative aneurysm rupture, thereby leading to a better neurological outcome after surgery. ${ }^{7}$ In premature aneurysm rupture, temporary clipping is a rescue step and a prerequisite for a better control of the intraoperative situation. According to the literature, the premature aneurysm rupture rate during surgery is between $6.6 \%$ and $35 \% .^{28,29}$ The rate of intraoperative aneurysm rupture in our patient population was $27 \%$, which is in line with rates reported in the literature.

Temporary clipping, either elective or as a rescue procedure, has been experimentally proven to induce changes in the vessel wall..$^{20,22}$ Thus, it was our hypothesis that temporary clipping is associated with a higher rate of TCD- vasospasm and/or DIND and/or DCI. The major finding of the present study was that temporary clipping during aneurysm surgery in patients with aSAH does not result in a higher incidence of TCD-vasospasm, DIND, or DCI, compared with cases in which temporary clipping was not needed.

Since elective and rescue temporary clipping are used in 2 different intraoperative situations possibly carrying different levels of risk for vasospasm and DCI, we evaluated its impact on TCD-vasospasm, DIND, and DCI separately. In comparison with elective temporary clipping, rescue temporary clipping is not associated with a significantly higher rate of TCD-vasospasm or DIND. However, DCI occurred significantly more often after rescue temporary clipping. Even in experienced hands and after temporary clipping, premature aneurysm rupture, especially if occurring prior to complete exposure of the aneurysm, might represent a poorly controlled surgical situation, necessitating a longer period of parent vessel occlusion for definitive aneurysm clipping and thereby increasing the risk of DCI.

The findings of our study are in contrast to those of Woertgen et al. ${ }^{34}$ who reviewed a database of 292 cases in which aSAH patients underwent aneurysm clipping. In their study, temporary vessel occlusion during aneurysm clipping aggravated vasospasm and increased the number of ischemic lesions. Furthermore, longer temporary occlusion time was related to poorer outcome. ${ }^{32}$ Surgery was performed at a mean of 3.9 days after aSAH (in contrast to our study population undergoing surgery at a mean of 2 days after aSAH). Thus, a considerable proportion of their patients were operated on at the beginning of the vasospasm phase, in which the vasospastic effect of temporary clipping might be substantially more pronounced than in earlier surgery. While it seems logical that longer temporary clipping time is related to hypoperfusion of the corresponding vessel territory, the role of the length of temporary clipping on the incidence of DCI in patients with aSAH who undergo aneurysm clipping still remains unclear. Unfortunately, the duration of temporary clipping was not recorded for all patients in our study, precluding a meaningful statistical analysis. In previous studies, the cutoff values for a safe duration of temporary clipping ranged from 10 to 20 minutes, without differentiation between elective and rescue temporary clipping. ${ }^{23,26,34}$

Two retrospective clinical studies support our findings. The effect of temporary artery occlusion during microsurgical clipping on patient outcome was evaluated in 382 patients with ruptured aneurysms and 246 patients with unruptured aneurysms. Temporary clipping with a mean duration of 19 minutes had no negative influence on clinical outcome. ${ }^{11,12}$ It is well known that vasospasm and DCI are the main contributors to poor outcome in aSAH patients. Despite not being investigated in these 2 studies, the lack of effect of temporary clipping on outcome can be seen as a surrogate parameter for a lack of effect of temporary clipping on the incidence of vasospasm and DCI.

\section{Strengths and Limitations of the Study}

The major strength of our study is the large number of patients included in the analysis. The major limitation is 
that the study was a retrospective analysis of partly prospectively, partly retrospectively collected data. Due to the retrospective nature of the study, the length of time for temporary clipping per vessel was not recorded consistently. Therefore, we could not analyze the impact of the time length of temporary clipping on the occurrence of DCI. One might consider the use of TCD sonography instead of angiography for detection of vasospasm as being a limitation. ${ }^{9,21}$ However, TCD sonography is a well-established noninvasive bedside monitoring technique with a likewise well-established cutoff value of $120 \mathrm{~cm} / \mathrm{sec}$ blood flow velocity being indicative for vasospasm.6,21,24 The limited repeatability of angiography due to its invasiveness might contribute to an underestimation of the incidence of vasospasm. Finally, we evaluated not only the incidence of TCD-vasospasm but also the incidence of DIND and DCI, which are parameters directly linked to vasospasm. Since treatment-related infarctions were ruled out by performing a postoperative CT scan, the infarctions that were considered in this study can be ascribed to delayed cerebral ischemia. The fact that we did not see divergent results for TCD-vasospasm, DIND, and DCI also supports the decision for vasospasm monitoring by TCD sonography. Conventional angiography was not performed in every patient to diagnose angiographic vasospasm. Consequently, we could not evaluate whether vasospasm occurred in the temporarily clipped vessels.

\section{Conclusions}

Temporary clipping does not increase the incidence of TCD-vasospasm, DIND, or DCI. There is no need to refrain from temporary clipping if it is deemed appropriate for successful management of a ruptured aneurysm.

\section{References}

1. Baldwin ME, Macdonald RL, Huo D, Novakovic RL, Goldenberg FD, Frank JI, et al: Early vasospasm on admission angiography in patients with aneurysmal subarachnoid hemorrhage is a predictor for in-hospital complications and poor outcome. Stroke 35:2506-2511, 2004 (Erratum in Stroke 36:175, 2005)

2. Charpentier C, Audibert G, Guillemin F, Civit T, Ducrocq X, Bracard S, et al: Multivariate analysis of predictors of cerebral vasospasm occurrence after aneurysmal subarachnoid hemorrhage. Stroke 30:1402-1408, 1999

3. Ciurea AV, Palade C, Voinescu D, Nica DA: Subarachnoid hemorrhage and cerebral vasospasm-literature review. J Med Life 6:120-125, 2013

4. Cooper JA, Tichauer KM, Boulton M, Elliott J, Diop M, Arango M, et al: Continuous monitoring of absolute cerebral blood flow by near-infrared spectroscopy during global and focal temporary vessel occlusion. J Appl Physiol (1985) 110:1691-1698, 2011

5. Danura H, Schatlo B, Marbacher S, Kerkeni H, Diepers M, Remonda L, et al: Acute angiographic vasospasm and the incidence of delayed cerebral vasospasm: preliminary results. Acta Neurochir Suppl 120:187-190, 2015

6. Deb S, Gogos AJ, Drummond KJ, Teddy PJ: The role of transcranial Doppler ultrasound monitoring in patients with aneurysmal subarachnoid haemorrhage. J Clin Neurosci 19:950-955, 2012

7. Dhandapani S, Pal SS, Gupta SK, Mohindra S, Chhabra R, Malhotra SK: Does the impact of elective temporary clip- ping on intraoperative rupture really influence neurological outcome after surgery for ruptured anterior circulation aneurysms?-A prospective multivariate study. Acta Neurochir (Wien) 155:237-246, 2013

8. Findlay JM, Macdonald RL, Weir BK, Grace MG: Surgical manipulation of primate cerebral arteries in established vasospasm. J Neurosurg 75:425-432, 1991

9. Frontera JA, Fernandez A, Schmidt JM, Claassen J, Wartenberg KE, Badjatia N, et al: Defining vasospasm after subarachnoid hemorrhage: what is the most clinically relevant definition? Stroke 40:1963-1968, 2009

10. Greenberg ED, Gobin YP, Riina H, Johnson CE, Tsiouris AJ, Comunale J, et al: Role of CT perfusion imaging in the diagnosis and treatment of vasospasm. Imaging Med 3:287-297, 2011

11. Griessenauer CJ, Poston TL, Shoja MM, Mortazavi MM, Falola M, Tubbs RS, et al: The impact of temporary artery occlusion during intracranial aneurysm surgery on long-term clinical outcome: part I. Patients with subarachnoid hemorrhage. World Neurosurg 82:140-148, 2014

12. Griessenauer CJ, Poston TL, Shoja MM, Mortazavi MM, Falola M, Tubbs RS, et al: The impact of temporary artery occlusion during intracranial aneurysm surgery on long-term clinical outcome: Part II. The patient who undergoes elective clipping. World Neurosurg 82:402-408, 2014

13. Gross BA, Rosalind Lai PM, Frerichs KU, Du R: Treatment modality and vasospasm after aneurysmal subarachnoid hemorrhage. World Neurosurg 82:e725-e730, 2014

14. Grunwald IQ, Kühn AL, Schmitt AJ, Balami JS: Aneurysmal SAH: current management and complications associated with treatment and disease. J Invasive Cardiol 26:30-37, 2014

15. Hoh BL, Topcuoglu MA, Singhal AB, Pryor JC, Rabinov JD, Rordorf GA, et al: Effect of clipping, craniotomy, or intravascular coiling on cerebral vasospasm and patient outcome after aneurysmal subarachnoid hemorrhage. Neurosurgery 55:779-789, 2004

16. Hosoda K, Fujita S, Kawaguchi T, Shose Y, Hamano S, Iwakura M: Effect of clot removal and surgical manipulation on regional cerebral blood flow and delayed vasospasm in early aneurysm surgery for subarachnoid hemorrhage. Surg Neurol 51:81-88, 1999

17. Jabbarli R, Reinhard M, Shah M, Roelz R, Niesen WD, Kaier $\mathrm{K}$, et al: Early vasospasm after aneurysmal subarachnoid hemorrhage predicts the occurrence and severity of symptomatic vasospasm and delayed cerebral ischemia. Cerebrovasc Dis 41:265-272, 2016

18. Kassell NF, Torner JC, Haley EC Jr, Jane JA, Adams HP, Kongable GL: The international cooperative study on the timing of aneurysm surgery. Part 1: Overall management results. J Neurosurg 73:18-36, 1990

19. Kassell NF, Torner JC, Jane JA, Haley EC Jr, Adams HP: The international cooperative study on the timing of aneurysm surgery. Part 2: Surgical results. J Neurosurg 73:37-47, 1990

20. Keskil S, Ceviker N, Baykaner K, Uluoğlu O, Ercan ZS: Early phase alterations in endothelium dependent vasorelaxation responses due to aneurysm clip application and related manipulations. Acta Neurochir (Wien) 139:71-76, 1997

21. Kühn AL, Balami JS, Grunwald IQ: Current management and treatment of cerebral vasospasm complicating SAH. CNS Neurol Disord Drug Targets 12:233-241, 2013

22. Kühnel TS, Müller GH: Experimental animal studies of clipinduced microvascular trauma. Microsurgery 24:241-247, 2004

23. Lavine SD, Masri LS, Levy ML, Giannotta SL: Temporary occlusion of the middle cerebral artery in intracranial aneurysm surgery: time limitation and advantage of brain protection. J Neurosurg 87:817-824, 1997

24. Macdonald RL, Wallace MC, Coyne TJ: The effect of sur- 
gery on the severity of vasospasm. J Neurosurg 80:433-439, 1994

25. Mills JN, Mehta V, Russin J, Amar AP, Rajamohan A, Mack WJ: Advanced imaging modalities in the detection of cerebral vasospasm. Neurol Res Int 2013:415960, 2013

26. Ogilvy CS, Carter BS, Kaplan S, Rich C, Crowell RM: Temporary vessel occlusion for aneurysm surgery: risk factors for stroke in patients protected by induced hypothermia and hypertension and intravenous mannitol administration. J Neurosurg 84:785-791, 1996

27. Pennings FA, Bouma GJ, Ince C: Direct observation of the human cerebral microcirculation during aneurysm surgery reveals increased arteriolar contractility. Stroke 35:12841288,2004

28. Sanelli PC, Jou A, Gold R, Reichman M, Greenberg E, John $\mathrm{M}$, et al: Using CT perfusion during the early baseline period in aneurysmal subarachnoid hemorrhage to assess for development of vasospasm. Neuroradiology 53:425-434, 2011

29. Sheth SA, Hausrath D, Numis AL, Lawton MT, Josephson SA: Intraoperative rerupture during surgical treatment of aneurysmal subarachnoid hemorrhage is not associated with an increased risk of vasospasm. J Neurosurg 120:409-414, 2014

30. Suzuki H, Taki W: Effect of aneurysm treatment modalities on cerebral vasospasm after aneurysmal subarachnoid hemorrhage. Acta Neurochir Suppl 115:99-105, 2013

31. Wachter D, Hans F, Kreitschmann-Andermahr I, Rohde V: Lower incidence of transcranial Doppler and symptomatic vasospasm after aneurysmal subarachnoid hemorrhage and aneurysm clipping in the elderly patient? Neurosurgery 69:261-267, 2011
32. Wachter D, Kreitschmann-Andermahr I, Gilsbach JM, Rohde V: Early surgery of multiple versus single aneurysms after subarachnoid hemorrhage: an increased risk for cerebral vasospasm? J Neurosurg 114:935-941, 2011

33. Williams JR: The Declaration of Helsinki and public health. Bull World Health Organ 86:650-652, 2008

34. Woertgen C, Rothoerl RD, Albert R, Schebesch KM, Ullrich OW: Effects of temporary clipping during aneurysm surgery. Neurol Res 30:542-546, 2008

\section{Disclosures}

The authors report no conflict of interest concerning the materials or methods used in this study or the findings specified in this paper.

\section{Author Contributions}

Conception and design: Malinova, Rohde, Mielke. Acquisition of data: Malinova, Voit. Analysis and interpretation of data: Malinova, Schatlo, Voit. Drafting the article: Malinova. Critically revising the article: Rohde, Mielke. Reviewed submitted version of manuscript: Rohde, Mielke. Approved the final version of the manuscript on behalf of all authors: Malinova. Statistical analysis: Schatlo. Study supervision: Mielke.

\section{Correspondence}

Vesna Malinova, Department of Neurosurgery, Georg-AugustUniversity Göttingen, Robert-Koch-Strasse 40, Göttingen 37075, Germany.email: vesna.malinova@gmail.com. 